\title{
A Novel Co-existence Algorithm for Unlicensed Fixed Power Devices
}

\author{
Durga P. Satapathy ${ }^{1}$ (Sprint) and Jon M. Peha ${ }^{2}$ (Carnegie Mellon University)
}

\begin{abstract}
In unlicensed spectrum, any device can transmit without a license. Such spectrum has major benefits, but serious challenges must first be overcome. Foremost is the risk of drastic performance degradation due to a lack of incentive to conserve shared resources. Previous work has shown this problem for devices that transmit for longer duration than necessary. This paper demonstrates this problem for devices always transmitting at maximum power to improve throughput. For devices with fixed transmit powers, the problem is solved if devices defer transmission when received interference exceeds defined thresholds. We propose a co-existence algorithm designed to optimize system throughput when each of two devices can transmit at the maximum power allowed. We show device performance with current unlicensed band regulations is rarely optimal, and that the proposed algorithm is better.
\end{abstract}

\section{INTRODUCTION}

In unlicensed spectrum, any device is free to transmit without a license that implies exclusive access. The Industry, Science and Medicine (ISM) bands have long been unlicensed, although most spectrum has traditionally been licensed [1]. The Federal Communications Commission (FCC) has of late increased unlicensed allocations, creating the Unlicensed Personal Communication Services (UPCS) band [2], the Unlicensed National Information Infrastructure (UNII) band [3], and the Millimeter Wave band [4]. The UPCS band is governed by a Spectrum Etiquette (known as the UPCS etiquette) [2,5], which is a set of rules regulating access to spectrum and its usage. Unlicensed spectrum has several benefits. It facilitates mobility of wireless applications, as no licenses are needed for new locations. It promotes spectrum sharing (as any device can transmit while others are idle,) and furthers experimentation and innovation. Three challenges must be overcome to realize such benefits. First, there may be mutual interference, as devices can transmit at will.

Second, enforcing efficient utilization is difficult as applications using unlicensed bands may vary greatly. Third, there is little inherent incentive to conserve shared spectrum. Thus, designers may adopt a greedy approach, where the more a device wastes shared spectrum to improve its performance, the more it is greedy. If this is common, the shared resource will be of little use. This phenomenon, referred to as a Tragedy of the Commons [6], made the Citizen Band radio service unusable in crowded regions, where users wasted spectrum with high-power transmitters. As the resources consumed by a device depend on transmission duration, bandwidth, and power, it may be greedy in any of these dimensions.

\footnotetext{
1d.p.satapathy@ieee.org; www.contrib.andrew.cmu.edu/usr/dsaq/home.html ${ }^{2}$ Associate Professor; Carnegie Mellon University; peha@ece.cmu.edu; http://www.ece.cmu.edu/ peha
}

Previous work $[7,8]$ has shown that greed in transmission duration can result in poor utilization of unlicensed spectrum. In this paper we demonstrate the same problem due to greed in the power dimension. Although all unlicensed bands enforce power limits to reduce interference, without any incentive to reduce power below the limit, greedy devices may transmit at the maximum power allowed to improve performance. Given information (such as power, offered load, and distance) about other devices sharing spectrum, the parameters that maximize system throughput can be determined. Without such explicit information, these parameters can only be chosen by an etiquette based on available information, e.g. local noise and received power. Etiquette design is complicated by the diversity of devices. Some devices can vary transmission power, and some cannot. Also, the power limits can vary from device to device.

For devices that transmit at fixed powers, we develop the Deferring etiquette that avoids a Tragedy of the Commons by requiring devices to defer transmission when the received interference exceeds defined thresholds. This etiquette optimizes system throughput as well as device throughput when two devices that can transmit at the maximum power allowed share spectrum. It also prevents starvation, which occurs if a given device can never transmit while another is transmitting, whereas the other device can always transmit. We use the following approach to compare the performance of devices: We assume that devices are designed to transmit at powers that maximize individual device throughput. We identify the powers at which devices reach equilibrium, and compare both system and individual device throughputs at each equilibrium with the optimal throughput and that without an etiquette. We show that system performance can be improved by discriminating between devices based on transmission power, and by creating multiple unlicensed bands such that each band caters to devices with a different range of power limits.

Section 2 presents our model to analyze greed in transmission power. Section 3 covers performance in unlicensed bands without an etiquette. Section 4 defines optimal performance of two devices sharing spectrum. Sections 5 and 6 discuss the UPCS and the Deferring etiquette respectively. Section 7 compares performance of existing and proposed etiquettes. Section 8 presents our conclusions.

\section{THE MODEL SCENARIO}

Our model has two wireless networks, each with two cooperating elements: a device and its basestation. Elements 
belonging to different networks do not cooperate. The networks share a single channel of fixed bandwidth $\mathrm{B}$, as in one-way systems and in either the uplink or the downlink of two-way systems (e.g. a wireless PBX). Without loss of generality, we consider devices transmitting to their basestations on this channel. The path loss between Device $i$ and Basestation $i$ is given by the propagation factor $\beta_{i}$, and the path loss between Basestation $i$ and Device $j$ is given by the propagation factor $\alpha_{i}$. We assume symmetry in propagation loss from one network to another is, i.e. $\alpha_{i}=\alpha_{j}=\alpha$. Since there will be devices in unlicensed bands that cannot determine the noise and the propagation factor, designers of etiquettes and access protocols must use values expected to be typical. Thus, the etiquettes function as if each network has the same noise $\mathrm{N}$, and $\beta_{i}=\beta_{j}=\beta$, where

$\beta$ is the propagation factor based on the anticipated path loss. The propagation factors $\alpha$ (or $\beta$ ) decrease with distance as dictated by the path loss model. Device $i$ transmits at power $0 \leq P_{i} \leq \gamma_{i} P_{\max }$, where $P_{\max }$ denotes the maximum transmission power allowed on the channel, and $\gamma_{i} P_{\max }$ denotes the power limit of Device $i$. With Device $j$ transmitting at power $P_{j}$, Basestation $i$ receives power $R_{i}=N+\alpha P_{j}$. We assume that Device i either has information about or can reasonably estimate the received power $R_{i}=N+\alpha P_{j}$. Thus, the signal-to-noise ratio (SNR) at Basestation $i$ is $\phi_{i}=\beta P_{i} /\left(N+\alpha P_{j}\right)$, and Basestation $j$ has $\phi_{j}=\beta P_{j} /\left(N+\alpha P_{i}\right)$. We define $\phi_{S}=\beta P_{\max } / N$ to be the SNR for Device $i$ when it transmits in isolation at power $P_{\max }$. We assume each Device $i$ has message error probability $E_{i}=\exp \left(-c \phi_{i}\right)$ where $c$ is a constant, as is appropriate for DPSK (Differential Phase Shift Keying) or non-coherent FSK (Frequency Shift Keying) modulation. Device $i$ has offered load $G_{i}$ which is the sum of the loads from arriving and retransmitted messages. Device $i$ has throughput $S_{i}=G_{i}\left(1-E_{i}\right)=G_{i}\left(1-\exp \left(-c \beta P_{i} /\left(N+\alpha P_{j}\right)\right)\right.$.

We determine the performance of devices by observing device throughput and system throughput as devices vary transmission parameters. These parameters are powers $P_{1}$ and $P_{2}$, power limits $\gamma_{1} P_{\max }$ and $\gamma_{2} P_{\max }$, loads $G_{1}$ and $G_{2}$, and the propagation factor $\alpha$. We observe the impact of varying these input parameters on throughput $S_{1}$, throughput $S_{2}$, and system throughput $S_{1}+S_{2}$, which are the output parameters for our model.

\section{UNLICENSED BANDS WITH NO ETIQUETTE}

Consider Device $i$ and Device $j$ that choose transmission power and load to maximize individual throughput. From
Theorem 1, if transmission powers $P_{i}$ and $P_{j}$ are fixed, devices maximize throughputs at $G_{i}=G_{j}=1$, i.e., by transmitting all the time. From Theorem 2, if $G_{i}=G_{j}=1$, devices maximize throughputs by transmitting at maximum power. Thus, devices would always transmit at maximum powers, and that is the only equilibrium. The device with higher power gets greater throughput. However, such greed can result in a Tragedy of the Commons. As the path loss between a device and its basestation increases, device performance gets worse. In scenarios where the path loss between each device and its basestation is large relative to the path loss between its basestation and the interfering device, device throughput can degrade drastically.

\section{OPTIMAL SYSTEM THROUGHPUT}

We now discuss the case where devices set their powers and offered loads to maximize system throughput, given each device has information about the transmission power, load and distance of the other device. This may not be practical, but it is a standard by which real etiquettes can be judged.

We first show that throughput is optimized when both devices always transmit. To maximize throughput, either both devices should transmit, or only the device with higher power should transmit. We accommodate both possibilities by assuming that both devices always transmit, but allow the power (and thus throughput) of either device to fall to zero. Equation 1 shows the derivative of system throughput with respect to $P_{1}$, with $P_{2}$ constant. That derivative is always positive when $P_{1}=0$, which shows that $P_{1}>0$ when throughput is maximized. By symmetry, the same is true of $P_{2}$.

$$
\begin{gathered}
d\left(S_{1}+S_{2}\right) / d P_{1}=c \beta /\left(N+\alpha P_{2}\right) \exp \left(-c \beta P_{1} /\left(N+\alpha P_{2}\right)\right) \\
-\left(c \beta \alpha P_{2} /\left(N+\alpha P_{1}\right)^{2}\right) \exp \left(-c \beta P_{2} /\left(N+\alpha P_{1}\right)\right)
\end{gathered}
$$

It is optimal for at least one device to transmit at maximum power, as shown by Theorem 3. It is always optimal for both devices to transmit at maximum power when the received interference is negligible. However, it is not always optimal for both devices to transmit at maximum powers. We have observed the optimal behavior to occur in two modes, one at small $\alpha$ and the other at large $\alpha$. We define the boundary between the regions to be $\alpha_{B}$. $\alpha_{B}$ varies with the power limits of devices.

Optimal Behavior: Without loss of generality, let devices have power limits $\gamma_{1}$ ? $\gamma_{2}$. When $\alpha<\alpha_{B}$, both devices transmit at maximum power. When $\alpha$ ? $\alpha_{B}$, Device 1 transmits at $\gamma_{1} P_{\max }$ and the other transmits at a lesser power $0 \leq P<\gamma_{2} P_{\max }$ where $d \alpha_{B} / d N>0$ and $d \alpha_{B} / d \gamma_{2}<0$. For $\gamma_{1}=\gamma_{2}$ and $N=0$, the boundary is given by $\alpha_{B}=c \beta$. 
It can be demonstrated that this behavior is optimal as follows. Theorems 4 and 5 together show that it is optimal for devices with equal power limits to transmit at maximum power as long as $\alpha \leq \alpha_{B}$. Theorem 4 shows it is optimal for devices to transmit at equal powers for all $0<\alpha / \beta \leq c$ when noise $N=0$, regardless of power limits. Transmitting at equal powers minimizes system throughput for all $\alpha / \beta>c$, i.e., it becomes optimal for devices to transmit at unequal powers. Theorem 5 shows that in a system with noise $N>0$, devices transmitting at equal power optimize system throughput at maximum power. The same phenomenon occurs with greater noise, except that the threshold at which optimal device behavior changes increases with noise. Indeed, with infinite noise, it is optimal for both devices to always transmit at maximum power, i.e., $\alpha_{B}=1$.

For devices with unequal power limits, Theorem 6 shows that regardless of $\alpha$, it is optimal for the device with higher power limit to transmit at a power greater than that of the other device. Thus, there is an inherent tradeoff between maximizing throughput and fairness for devices with unequal power limits.

\section{THE UPCS ETIQUETTE}

The UPCS etiquette specifies an upper power limit $P_{\max }=100 \sqrt{B} \mathrm{~mW}$, where $B$ is the bandwidth in MHz. The etiquette enforces a Listen Before Talk (LBT) rule, requiring devices to transmit only if the received power is below a threshold throughout a specified monitoring period. It also allows devices to increase the LBT threshold by a dB for each $\mathrm{dB}$ reduction from the maximum power allowed.

With the UPCS etiquette, there are three ranges of $\alpha$ that characterize devices that transmit at fixed powers. The first is $\alpha$ for which each device receives power below its LBT threshold and always transmits, as specified by Theorem 7 . The second exists only for devices with unequal powers, for which the higher power device starves as shown by Theorem 8 . For all other $\alpha$, only one device transmits at a time.

\section{THE DEFERRING ETIQUETTE}

The Deferring etiquette attempts to maximize system throughput when each device is designed to either transmit at a fixed power or defer transmission to other devices. This etiquette optimizes system throughput as well as individual device throughput when two such devices that can transmit at power $P_{\max }$ (the maximum power allowed on a channel) share spectrum. This is significant because individual device throughput in unlicensed bands with no etiquette is maximized when devices always transmit at maximum power, as shown in Section 3. It is also desirable that the etiquette provide adequate performance for the case of one or more devices with $\gamma<1$. There are two design principles to be met by the Deferring etiquette. First, the system throughput must be optimal when each device can transmit at $P_{\max }$. Second, no device should face the risk of starvation.

Theorem 9 shows that when each device transmits at $P_{\max }$, system throughput is optimized if each device defers transmission when received power exceeds threshold $N+\alpha_{D} P_{\max }$, where $\alpha_{D}$ is specified by Equation 2. The Deferring etiquette is based on $\alpha_{D}$.

Deferring Etiquette: A Device $i$ with maximum transmission power $\gamma_{i} P_{\max }$ must defer transmission when received power is greater than a threshold $T_{i}=N+\alpha_{D} P_{\max } / \gamma_{i}$, where $N$ is the local noise, $\gamma_{i} P_{\max }$ is Device $i$ s power limit and $\alpha_{D}$ is given by the solution of $\exp \left(-c \beta P_{\max } /\left(N+\alpha_{D} P_{\max }\right)\right)-0.5+0.5 \exp \left(-c \beta P_{\max } / N\right)=0$

The Deferring etiquette meets both design criteria. Devices transmitting at $P_{\max }$ optimize system throughput as they defer at the optimal threshold. Theorem 10 shows that at any given $\alpha$, if any device receives power above its threshold, so does the other. Provided that some mechanism prevents devices from transmitting indefinitely [8], devices will alternate transmissions. Thus, starvation is not a problem with the Deferring etiquette. When devices take turns transmitting, the system throughput is maximized with $G_{1}+G_{2}=1$. When devices alternate transmissions, we assume devices follow a fairness criteria with $G_{1}=G_{2}=0.5$.

Two regions of $\alpha$ characterize behavior of devices that maximize individual device throughput with the Deferring etiquette, as given by Theorem 11. When $\alpha$ is small such that each device receives power below its threshold, devices transmit at maximum powers. At greater $\alpha$, devices reach equilibrium at which one device transmits at maximum power and the other does not transmit at all. Let $\alpha_{\text {opt }}$ represent $\alpha$ at which system throughput is optimized when devices alternate transmissions for any given $\gamma_{1} \leq 1$ and $\gamma_{2} \leq 1$. Theorem 12 shows that $\alpha_{\text {etq }}$ ? $\alpha_{\text {opt }}$, i.e. devices defer at $\alpha$ that is equal to or greater than the optimal. Thus, the Deferring etiquette is optimal not only when devices transmit at $P_{\max }$, but also whenever $\alpha_{o p t}>1$, i.e. when devices optimally never defer (e.g. when both devices have low power limits such that mutual interference is negligible, or when one device has a power limit significantly lower than the other).

The system throughput is sub-optimal for medium ranges of $\gamma$, for which the etiquette is not designed. As devices defer at $\alpha_{\text {etq }}$ ? $\alpha_{\text {opt }}$, the system throughput is sub-optimal in the range $\alpha_{\text {opt }}<\alpha<\alpha_{\text {etq }}$ only. Whenever the system 


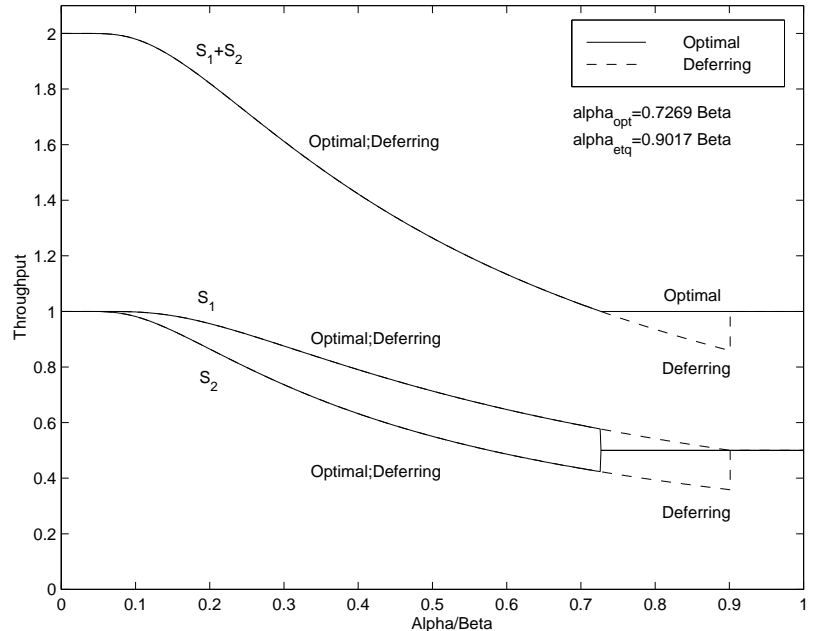

Figure 1: System throughput (with the Deferring etiquette and optimal ) vs. propagation factor ratio $\alpha / \beta \cdot \gamma_{1}=1$ and $\gamma_{2}=0.8$ with each device at maximum power. When devices alternate transmissions, $G_{1}=G_{2}=0.5$.

throughput at equilibrium is sub-optimal, low-power devices have a lower throughput with the Deferring etiquette as compared to the optimal, and high-power devices have a higher throughput with the Deferring etiquette, as shown in Figure 1. Furthermore, the system throughput is optimal or near-optimal when power limits are high, with performance being increasingly suboptimal as power limits decrease from high to medium values. These observations indicate that system performance with the Deferring etiquette would improve with multiple unlicensed bands, each catering to devices with a smaller range of power limits, instead of a single band for devices with a wide range of power limits.

\section{PERFORMANCE COMPARISON}

In this section we compare performance of devices with the Deferring etiquette, the UPCS etiquette, and with no etiquette by evaluating the throughputs of devices at equilibrium. As shown in Appendix B, the relative performance of etiquettes depends on the parameter $\sqrt{B} / \beta$. We therefore select various values of $\sqrt{B} / \beta$ in a $100 \mathrm{MHz}$ band for etiquette comparison. We first address devices with equal power limits. For devices transmitting at fixed powers with $\gamma_{1}=\gamma_{2}=1$, the Deferring etiquette results in optimal system throughput, whereas performance without an etiquette is suboptimal. For a given channel bandwidth, the UPCS etiquette performance is optimal only for a specific $\beta$, whereas the

Deferring etiquette does not suffer from this constraint. Figure 2 shows these results, with the UPCS etiquette performance is optimal only with $\beta=8.74 \leftrightarrow 10^{-10}$.

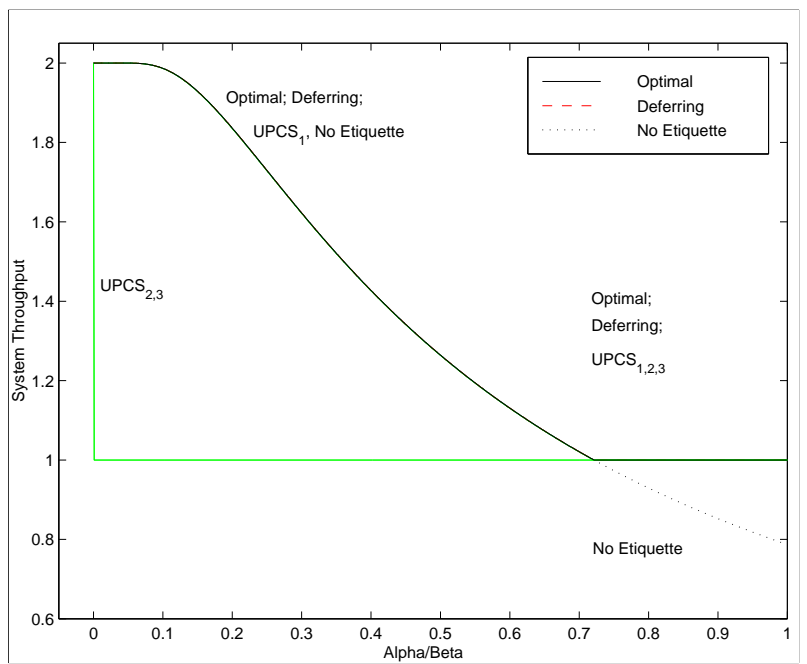

Figure 2: System throughputs vs. propagation factor ratio $\alpha / \beta$ with the Deferring etiquette, the UPCS etiquette, with no etiquette and the optimal case for devices transmitting at fixed powers with power limit parameters $\gamma_{1}=\gamma_{2}=1$ for a $100 \mathrm{MHz}$ UNII band with $N / P_{\max }=4 \leftrightarrow 0^{-13}$. The UPCS etiquette is shown with $\beta=8.74 \leftrightarrow 0^{-10}$ as $\mathrm{UPCS}_{1}, \beta=10^{-6}$ as $\mathrm{UPCS}_{2}$ and $\beta=1$ as $\mathrm{UPCS}_{3}$.

For $\gamma_{1}=\gamma_{2}=\gamma<1$, performance is sub-optimal for all cases. Performance with all but the UPCS etiquette is as good or better than that with no etiquette. Devices defer at $\alpha_{\text {etq }}>\alpha_{\text {opt }}$ with the Deferring etiquette. Performance with the UPCS etiquette is identical to that of the Deferring etiquette for $\sqrt{B} / \beta$ at which $\alpha_{U P C S}=\alpha_{D}$. Figure 3 shows these results for $\gamma_{1}=\gamma_{2}=0.9$ at $N / P_{\max }=4 \leftrightarrow 10^{-13}$. With $\beta=8.74 \leftrightarrow 0^{-10}$,

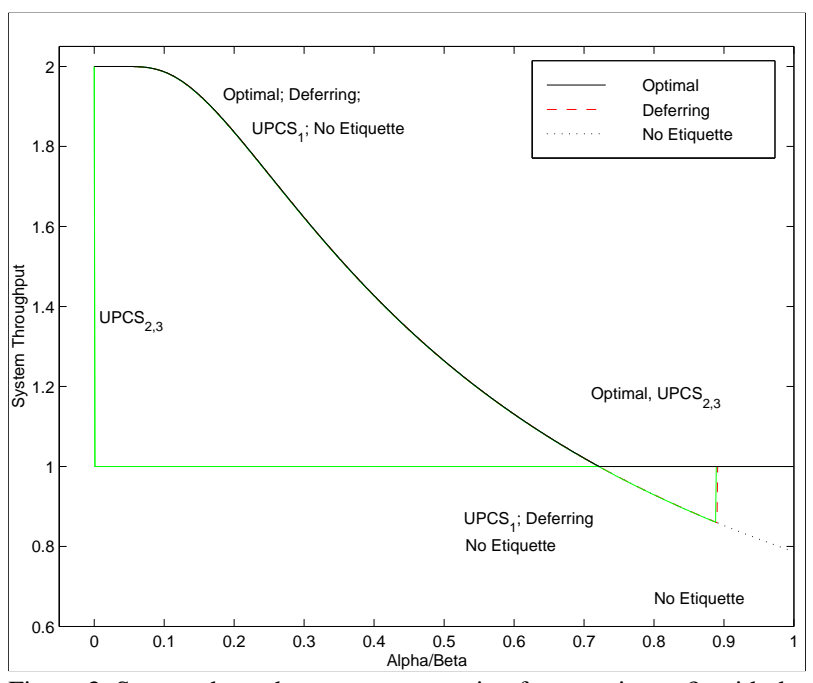

Figure 3: System throughputs vs. propagation factor ratio $\alpha / \beta$ with the Deferring etiquette, the UPCS etiquette, with no etiquette and the optimal case for devices transmitting at fixed powers with power limit parameters $\gamma_{1}=\gamma_{2}=0.9$ for a $100 \mathrm{MHz}$ UNII band with $N / P_{\max }=4 \leftrightarrow 0^{-13}$. The UPCS etiquette is shown with $\beta=8.74 \leftrightarrow \mathrm{H}^{-10}$ as $\mathrm{UPCS}_{1}, \beta=10^{-6}$ as $\mathrm{UPCS}_{2}$ and $\beta=1$ as $\mathrm{UPCS}_{3}$. 
the UPCS etiquette performance is exactly the same as with the Deferring etiquette. For the specific $\beta$ at which

$\alpha_{U P C S}=\alpha_{\text {opt }}$, the UPCS etiquette performance is optimal, as devices then defer at the optimal threshold.

For devices with unequal transmission powers, devices never starve with the Deferring etiquette. The UPCS etiquette may lead to starvation for devices with fixed unequal powers, as shown in Theorem 8.

In summary, only the Deferring etiquette is optimal when $\gamma_{1}=\gamma_{2}=1$ and it is superior to the others for $\gamma$ close to 1 . Performance with the UPCS etiquette varies widely with $\beta$ and can be far from optimal. Furthermore, performance with the Deferring etiquette is always as good or better than that with no etiquette. Thus, we find the Deferring etiquette most favorable for devices with fixed transmission powers.

\section{CONCLUSION}

Unlicensed spectrum has several advantages. However, as individual devices have little inherent incentive to conserve spectrum, they may hoard shared resources to improve their performance. Thus, designers may adopt a greedy approach, where the more a device wastes shared spectrum to improve its performance, the more it is greedy. Devices may be greedy in consuming any of these resources: transmission duration, bandwidth, and transmission power. Previous work has shown severe performance degradation due to greed in transmission duration, and suggested solutions. This paper explores greed in transmission power. We show that in bands with only power limits, devices would maximize throughput by always transmitting at maximum power, which also optimizes system throughput when devices are far apart. When devices are near, transmitting at maximum power leads to suboptimal performance and reduced frequency reuse. This problem can be solved by a properly designed etiquette (i.e. a set of rules regulating spectrum resource usage). We propose the Deferring etiquette that has been designed to optimize individual throughput for devices in isolation, and is optimal when each of two fixed power devices can transmit at the maximum power allowed. This etiquette requires devices to defer transmission when the received interference reaches a threshold. It also prevents starvation, which occurs if a given device can never transmit while another is transmitting, whereas the other device can always transmit. We present analysis to demonstrate that this etiquette offers better performance than the current unlicensed band regulations. We show that system performance can be improved by discriminating between devices based on transmission power, and by creating multiple unlicensed bands such that each band caters to devices with a different range of power limits.

\section{APPENDIX A}

Theorem 1: If $P_{i}, P_{j}$ and $G_{j}$ are fixed, and $0 \leq G_{i} \leq 1$, then $S_{i}$ is maximized at $G_{i}=1$.

Proof: Device $i$ has throughput $S_{i}=G_{i}\left(1-E_{i}\right)$ where $G_{i}$ is its offered load, and $E_{i}$ is its message error rate given by $E_{i}=G_{j} \exp \left(-c \beta P_{i} /\left(N+\alpha P_{j}\right)\right)+\left(1-G_{j}\right) \exp \left(-c \beta P_{i} / N\right)$. As $d S_{i} / d G_{i}=\left(1-E_{i}\right)>0, S_{i}$ is maximized at $G_{i}=1$.

Theorem 2: If $G_{i}, G_{j}$ and $P_{j}$ are fixed, and $0 \leq G_{j} \leq 1$, then $S_{i}$ is maximized at $P_{i}=P_{\max }$.

Proof: As $S_{i}=G_{i}\left(1-E_{i}\right), d S_{i} / d P_{i}=-G_{i} d E_{i} / d P_{i}$ $d E_{i} / d P_{i}=-\left(\mathrm{c} \beta G_{j} /\left(\mathrm{N}+\alpha P_{j}\right)\right) \exp \left(-c \beta P_{i} /\left(N+\alpha P_{j}\right)\right)$ $-\left(c \beta G_{j} / N\right) \exp \left(-c \beta P_{1} / N\right)<0$

Thus, $d S_{i} / d P_{i}>0$ and $S_{i}$ is maximized at $P_{i}=P_{\max }$.

Theorem 3: At least one device must transmit at maximum power for system throughput to be optimal.

Proof by contradiction: From Theorem 1, devices maximize individual throughputs with $G_{1}=G_{2}=1$. Assume system throughput is optimal when devices transmit at powers $P_{1}<\gamma_{1} P_{\max }$ and $P_{2}<\gamma_{2} P_{\max }$. Device throughputs are $S_{1}=1-E_{1}=1-\exp \left(-c \phi_{1}\right)$ and $S_{2}=1-E_{2}=1-\exp \left(-c \phi_{2}\right)$ where $\phi_{1}=\beta P_{1} /\left(N+\alpha P_{2}\right)$ and $\phi_{2}=\beta P_{2} /\left(N+\alpha P_{1}\right)$. Without loss of generality, let devices be numbered such that $P_{1}$ ? $P_{2}$. Thus, $\alpha \phi_{2}=\alpha \beta P_{2} /\left(N+\alpha P_{1}\right) \leq \alpha \beta P_{1} /\left(N+\alpha P_{1}\right) \leq 1$ as $0 \leq \beta \leq 1$. As $\phi_{1}=\beta P_{1} /\left(N+\alpha P_{2}\right)=\beta /\left(\left(N+\alpha N \phi_{2} / \beta\right) / P_{1}+\alpha^{2} \phi_{2} / \beta\right)$, increasing $P_{1}$ and $P_{2}$ such that $\phi_{2}$ remains constant results in an increase in $\phi_{1}$ and therefore in $S_{1}$ while $S_{2}$ stays the same. Thus, the assumption that system throughput is optimal at powers $P_{1}<\gamma_{1} P_{\max }$ and $P_{2}<\gamma_{2} P_{\max }$ is false.

Theorem 4: When $N=0$, the system throughput is maximized when both devices transmit at equal power for $0 \leq \alpha / \beta \leq c$ and at unequal powers for all $\alpha / \beta>c$.

Proof: From Theorem 1, devices maximize individual throughputs with $G_{1}=G_{2}=1$. Thus, the system throughput is $S_{1}+S_{2}=2-\exp \left(-c \beta P_{1} /\left(N+\alpha P_{2}\right)\right)-\exp \left(-c \beta P_{2} /\left(N+\alpha P_{1}\right)\right)$ With $N=0, S_{1}+S_{2}=2-\exp \left(-c \beta P_{1} / \alpha P_{2}\right)-\exp \left(-c \beta P_{2} / \alpha P_{1}\right)$ $S_{1}+S_{2}=2-\exp (-\eta x)-\exp (-\eta / x)$ where $\eta=c \beta / \alpha$ and $x=P_{1} / P_{2}$ Thus, $d\left(S_{1}+S_{2}\right) / d x=\eta \exp (-\eta x)-\left(\eta / x^{2}\right) \exp (-\eta / x)$.

$d\left(S_{1}+S_{2}\right) /\left.d x\right|_{x=1}=0$ and $d^{2}\left(S_{1}+S_{2}\right) /\left.d x^{2}\right|_{x=1}=\eta \exp (-\eta)(\eta-1)$.

Thus, $x=1$ (i.e. $P_{1}=P_{2}$ ) maximizes $S_{1}+S_{2}$ $\forall \eta=(c \beta / \alpha)<1$ and minimizes $S_{1}+S_{2} \forall \eta=(c \beta / \alpha)>1$.

Theorem 5: When devices transmit simultaneously at equal powers and noise $N>0$, system throughput is maximized when they transmit at maximum power. 
Proof: Device 1 and 2 transmit at power $P$ with loads

$G_{1}=G_{2}=1$. The system throughput is given by

$S_{1}+S_{2}=2[1-\exp (-c \beta P /(N+\alpha P))]$.

$d\left(S_{1}+S_{2}\right) / d P=c \beta N /(N+\alpha P)^{2} \exp (-c \beta P /(N+\alpha P)>0$.

Thus, system throughput is maximized when $P=P_{\max }$. Note this holds true only for $N>0$.

Theorem 6: When $\gamma_{1}>\gamma_{2}$, at least one of these conditions must be met when the system throughput is maximized: 1. $P_{1}=\gamma_{1} P_{\max }>\gamma_{2} P_{\max }$ ? $P_{2}$ 2. $\gamma_{1} P_{\max } ? P_{1}>\gamma_{2} P_{\max }=P_{2}$ Proof: From Theorem 3, at least one device transmits at maximum power when system throughput is optimized. If system throughput is optimal with Device 1 at maximum power, then $P_{1}=\gamma_{1} P_{\max }>\gamma_{2} P_{\max }$ ? $P_{2}$ (i.e. condition 1 is met). Consider the case where system throughput is optimal with Device 2 at $P_{2}=\gamma_{2} P_{\max }$. Assume $P_{1}=P<\gamma_{2} P_{\max }$ optimizes system throughput. System throughput remains the same if devices switch powers such that $P_{1}=\gamma_{2} P_{\max } ; P_{2}=P<\gamma_{2} P_{\max }$. As neither device is at maximum power, it follows from Theorem 3 that this is not optimal. Given system throughput is optimal with $P_{2}=\gamma_{2} P_{\max }$, Device 1 cannot have $P_{1}=P<\gamma_{2} P_{\max }$. From Equation 1, the derivative of system throughput with respect to $P_{1}$ is positive at $P_{1}=P_{2}$. Thus, when system throughput is optimal with Device 2 at $P_{2}=\gamma_{2} P_{\text {max }}$, Device 1 must have $P_{1}=P>\gamma_{2} P_{\text {max }}$, i.e. $\gamma_{1} P_{\max } ? P_{1}>\gamma_{2} P_{\max }=P_{2}$ (i.e. condition 2 must be met).

Theorem 7: For $\gamma_{1} ? \gamma_{2}$ and $\alpha<\left(K / \gamma_{1} P_{\max }-N\right) / \gamma_{2} P_{\max }$, each device receives power less than its LBT threshold, where $K \cong 1585 N P_{\text {max }}$.

Proof: For $P_{i} x \mathrm{~dB}$ below $P_{\text {max }}$, the LBT threshold is $T_{i}=32+x \mathrm{~dB}$ above noise $N$, i.e., $T_{i}=K / P_{i}$ where $K \cong 1585 N P_{\max }$. Equivalently, the maximum transmit power is $K / R_{i}$ where $R_{i}=N+\alpha P_{j}$. Device $i$ receives power below its threshold only for $\alpha$ at which $K / R_{i} ? \gamma_{i} P_{\max }$. With $P_{2}=\gamma_{2} P_{\max }, R_{1}=N+\alpha \gamma_{2} P_{\max }<K /\left(\gamma_{1} P_{\max }\right)$ for $0<\alpha<\left(K / \gamma_{1} P_{\max }-N\right) / \gamma_{2} P_{\max }$. Likewise, Device 2 receives power below its threshold only for $0<\alpha<\left(K / \gamma_{2} P_{\max }-N\right) / \gamma_{1} P_{\max }$. Thus, both devices receive power below their respective thresholds in the range $0<\alpha<\min \left\{\left(K / \gamma_{1} P_{\max }-N\right) / \gamma_{2} P_{\max },\left(K / \gamma_{2} P_{\max }-N\right) / \gamma_{1} P_{\max }\right\}$.As $\gamma_{1} ? \gamma_{2},\left(K / \gamma_{1} P_{\max }-N\right) / \gamma_{2} P_{\max } \leq\left(K / \gamma_{2} P_{\max }-N\right) / \gamma_{1} P_{\max }$. Thus, there is only one equilibrium $P_{1}=\gamma_{1} P_{\max }$ and $P_{2}=\gamma_{2} P_{\max }$ for $0<\alpha<\left(K / \gamma_{1} P_{\max }-N\right) / \gamma_{2} P_{\max }$.

Theorem 8: For $\left(K / \gamma_{2} P_{\max }-N\right) / \gamma_{1} P_{\max } \leq \alpha \leq\left(K / \gamma_{1} P_{\max }-N\right) / \gamma_{2} P_{\text {max }}$ with $P_{1}=\gamma_{1} P_{\text {max }}, P_{2}=\gamma_{2} P_{\text {max }}$ and $\gamma_{1}>\gamma_{2}$, Device 2 can transmit at will but Device 1 can only transmit when Device 2 is not.
Proof: Device 1 receives power below its threshold $K /\left(\gamma_{1} P_{\max }\right)$ only if $R_{1}=N+\alpha \gamma_{2} P_{\max }<K /\left(\gamma_{1} P_{\max }\right)$, i.e. for $0<\alpha<\left(K / \gamma_{1} P_{\max }-N\right) / \gamma_{2} P_{\max }$. Likewise, Device 2 receives power below threshold for $0<\alpha<\left(K / \gamma_{2} P_{\max }-N\right) / \gamma_{1} P_{\max }$. As $\gamma_{1}>\gamma_{2},\left(K / \gamma_{1} P_{\max }-N\right) / \gamma_{2} P_{\max }<\left(K / \gamma_{2} P_{\max }-N\right) / \gamma_{1} P_{\max }$. Thus, for $\left(K / \gamma_{2} P_{\max }-N\right) / \gamma_{1} P_{\max } \leq \alpha \leq\left(K / \gamma_{1} P_{\max }-N\right) / \gamma_{2} P_{\max }$, Device 2 can transmit even if Device 1 is transmitting, but Device 1 cannot transmit if Device 2 is transmitting.

Theorem 9: If $P_{1}=P_{2}=P_{\max }$, the system throughput is optimized if Device $i$ defers from transmission when the received power exceeds a threshold $T_{i}=N+\alpha_{D} P_{\max }$, where $N$ is the local noise and $\alpha_{D}$ is given by the equation $\exp \left(-\beta P_{\max } /\left(N+\alpha_{D} P_{\max }\right)\right)=0.5+0.5 \exp \left(-\beta P_{\max } / N\right)$.

Proof: Let power loss factor be $\alpha$. When $G_{1}=G_{2}=1$, $S_{1}+S_{2}=2-\exp \left(-c \beta P_{1} /\left(N+\alpha P_{2}\right)\right)-\exp \left(-c \beta P_{2} /\left(N+\alpha P_{1}\right)\right)$. With $P_{1}=P_{2}=P_{\text {max }}, S_{1}+S_{2}=2\left(1-\exp \left(-c \beta P_{\text {max }} /\left(N+\alpha P_{\text {max }}\right)\right)\right.$ which decreases monotonically as $\alpha$ increases. When devices take turns transmitting, the system throughput is maximized with $G_{1}+G_{2}=1$ and is $S_{1}+S_{2}=1-\exp \left(-c \beta P_{\max } / N\right)$. At optimal threshold, system throughput when devices share the channel reduces to the throughput when devices take turns transmitting, i.e. $\alpha_{D}=\alpha$ which satisfies

$2\left(1-\exp \left(-c \beta P_{\max } /\left(N+\alpha P_{\max }\right)\right)=1-\exp \left(-c \beta P_{\max } / N\right)\right.$, i.e., $\exp \left(-c \beta P_{\max } /\left(N+\alpha_{D} P_{\max }\right)\right)=0.5+0.5 \exp \left(-c \beta P_{\max } / N\right)$.

Theorem 10: For any given $\alpha$, the etiquette requires Device $i$ to defer transmission to Device $j$ when Device $j$ is transmitting if and only if Device $j$ is required to defer transmission to Device $i$ when Device $i$ is transmitting. Proof: Consider Device $i$ and Device $j$ transmitting at maximum powers $\gamma_{i} P_{\max }$ and $\gamma_{j} P_{\max }$ respectively. Let Device $i$ receive power equal to or exceeding its threshold at a given $\alpha$, i.e. $R_{i}=N+\alpha \gamma_{j} P_{\max } ? T_{i}=N+\alpha_{D} P_{\max } / \gamma_{i}$. Thus, $\alpha ? \alpha_{D} /\left(\gamma_{i} \gamma_{j}\right)$. Device $j$ receives power given by $R_{j}=N+\alpha \gamma_{i} P_{\max } \leq N+\alpha_{D} P_{\max } / \gamma_{j}=T_{j}$, i.e. Device $j$ does not receive power less than its threshold.

Theorem 11: With the Deferring etiquette, devices reach equilibrium at their maximum powers $P_{1}=\gamma_{1} P_{\max }$ and $P_{2}=\gamma_{2} P_{\max }$ for all $\alpha<\alpha_{e t q}$, where $\alpha_{e t q}=\alpha_{D} /\left(\gamma_{1} \gamma_{2}\right)$. For $\alpha>\alpha_{\text {etq }}$, there exist two equilibria given by $P_{1}=\gamma_{1} P_{\max } ; P_{2}=0$ and $P_{1}=0 ; P_{2}=\gamma_{2} P_{\max }$.

Proof: Device 1 transmits at $P_{1} \leq \gamma_{1} P_{\max }$ and has threshold $T_{1}=N+\alpha_{D} P_{\max } / \gamma_{1}$. Device 2 transmits at $P_{2} \leq \gamma_{2} P_{\max }$ and has threshold $T_{2}=N+\alpha_{D} P_{\text {max }} / \gamma_{2} \cdot R_{1}=N+\alpha P_{2}, R_{2}=N+\alpha P_{1}$.

For $\alpha<\alpha_{e t q}, R_{1}<N+\alpha_{D} P_{2} /\left(\gamma_{1} \gamma_{2}\right) \leq N+\alpha_{D} P_{\max } / \gamma_{1}=T_{1}$. For $\alpha<\alpha_{\text {etq }}, R_{2}<N+\alpha_{D} P_{1} /\left(\gamma_{1} \gamma_{2}\right) \leq N+\alpha_{D} P_{\max } / \gamma_{2}=T_{2}$. Thus, there is only one equilibrium $P_{1}=\gamma_{1} P_{\text {max }} ; P_{2}=\gamma_{2} P_{\text {max }}$ for all $\alpha<\alpha_{\text {etq }}$. 
For all $\alpha ? \alpha_{\text {etq }}$, if Device 2 is transmitting at equilibrium, As $R_{1}=N+\alpha \gamma_{2} P_{\max } ? N+\alpha_{D} P_{\max } / \gamma_{1}=T_{1}$, Device 1 does not transmit. Likewise, if Device 1 is transmitting at equilibrium, $R_{2}=N+\alpha \gamma_{1} P_{\max } ? N+\alpha_{D} P_{\max } / \gamma_{2}=T_{2}$ and Device 2 does not transmit. Thus, for all $\alpha ? \alpha_{\text {etq }}$, both devices cannot transmit simultaneously at equilibrium, i.e. there only exist two equilibria at which either device transmits at maximum power and the other is idle. These equilibria are given by $P_{1}=\gamma_{1} P_{\max } ; P_{2}=0$ and $P_{1}=0 ; P_{2}=\gamma_{2} P_{\max }$.

Theorem 12: $\alpha_{\text {etq }}$ ? $\alpha_{\text {opt }}$

Proof: We have $\alpha_{e t q}=\alpha_{D} /\left(\gamma_{1} \gamma_{2}\right)$, where $\alpha_{D}$ is given by $\exp \left(-c \beta P_{\max } /\left(N+\alpha_{D} P_{\max }\right)\right)=0.5+0.5 \exp \left(-c \beta P_{\max } / N\right)$ $\alpha_{\text {opt }}$ is given by the solution of

$$
\begin{aligned}
& \exp \left(\frac{-c \beta \gamma_{1} P_{\max }}{N+\alpha_{o p t} \gamma_{2} P_{\max }}\right)+\exp \left(\frac{-c \beta \gamma_{2} P_{\max }}{N+\alpha_{o p t} \gamma_{1} P_{\max }}\right) \\
& =\left[0.5+0.5 \exp \left(-c \beta \gamma_{1} P_{\max } / N\right)\right]+\left[0.5+0.5 \exp \left(-c \beta \gamma_{2} P_{\max } / N\right)\right]
\end{aligned}
$$

We will first show that

$\exp \left(-c \beta \gamma_{1} P_{\max } /\left(N+\alpha_{e t q} \gamma_{2} P_{\max }\right)\right) ?\left[0.5+0.5 \exp \left(-c \beta \gamma_{1} P_{\max } / N\right)\right]$.

$\exp \left(\frac{-c \beta \gamma_{1} P_{\max }}{N+\alpha_{\text {etq }} \gamma_{2} P_{\max }}\right)=\exp \left(\frac{-c \beta \gamma_{1} P_{\max }}{N+\alpha_{D} P_{\max } / \gamma_{1}}\right)$

$? \exp \left(-c \beta \gamma_{1} P_{\max } /\left(N+\alpha_{D} P_{\max }\right)\right)$ as $0<\gamma_{1} \leq 1$

$?(0.5+0.5 k)^{\gamma_{1}}$ by definition of $\alpha_{\mathrm{D}}$, where $k=\exp \left(-c \beta P_{\max } / N\right)$.

$\left[0.5+0.5 \exp \left(-c \beta \gamma_{1} P_{\max } / N\right)\right]=0.5+0.5 k^{\gamma_{1}}$.

As $k=\exp \left(-c \beta P_{\max } / N\right), 0 \leq k \leq 1$. Let

$f\left(k, \gamma_{1}\right)=(0.5+0.5 k)^{\gamma_{1}}-\left(0.5+0.5 k \gamma_{1}\right)$.

For $\gamma_{1}<1$ and $k=0, f\left(k, \gamma_{1}\right)=0.5^{\gamma_{1}}-0.5>0$. For $\gamma_{1}<1$ and $k<1$, $d f\left(k, \gamma_{1}\right) / d k=0.5 \gamma_{1}\left[(0.5+0.5 k)^{\gamma_{1}-1}-k^{\left(\gamma_{1}-1\right)}\right]$

$=0.5 \gamma_{1}\left[(1 /(0.5+0.5 k))^{1-\gamma_{1}}-(1 / k)^{1-\gamma_{1}}\right]<0$ as $k<1$. If $k=1$,

$f\left(k, \gamma_{1}\right)=0$. Thus, if $\gamma_{1}<1, f\left(k, \gamma_{1}\right) ? 0 \forall k$. If $\gamma_{1}=1$,

$f\left(k, \gamma_{1}\right)=0$. Thus, $\forall k, \gamma_{1}, f(k)$ ? 0 , i.e.,

$\exp \left(-c \beta \gamma_{1} P_{\max } /\left(N+\alpha_{e t q} \gamma_{2} P_{\max }\right)\right) ?\left[0.5+0.5 \exp \left(-c \beta \gamma_{1} P_{\max } / N\right)\right]$

By symmetry,

$\exp \left(-c \beta \gamma_{2} P_{\max } /\left(N+\alpha_{e t q} \gamma_{1} P_{\max }\right)\right) ?\left[0.5+0.5 \exp \left(-c \beta \gamma_{2} P_{\max } / N\right)\right]$

$\exp \left(\frac{-c \beta \gamma_{1} P_{\max }}{N+\alpha_{e t q} \gamma_{2} P_{\max }}\right)+\exp \left(\frac{-c \beta \gamma_{2} P_{\max }}{N+\alpha_{e t q} \gamma_{1} P_{\max }}\right)$

$?\left[0.5+0.5 \exp \left(-c \beta \gamma_{1} P_{\max } / N\right)+0.5+0.5 \exp \left(-c \beta \gamma_{2} P_{\max } / N\right)\right]$

$=\left[\exp \left(\frac{-c \beta \gamma_{1} P_{\max }}{N+\alpha_{\text {opt }} \gamma_{2} P_{\max }}\right)+\exp \left(\frac{-c \beta \gamma_{2} P_{\max }}{N+\alpha_{\text {opt }} \gamma_{1} P_{\max }}\right)\right]$

\section{APPENDIX B}

For $P_{i}=\gamma_{i} P_{\text {max }}$, the UPCS etiquette threshold is $T_{i} \cong 1585 N / \gamma_{i}$ with $N=k T B$, where $k$ is the Boltzman constant, $T$ is temperature in Kelvin, and $B$ is channel bandwidth in $\mathrm{Hz}$. For $\gamma_{i}=1, N+\alpha_{U P C S} P_{\max }=T_{i}$ i.e. $\alpha_{U P C S}=1584 N / P_{\max }$. With the UPCS etiquette, the maximum power allowed is $P_{\max }=10^{-4} \sqrt{B}$. Thus, $\alpha_{U P C S}=1584 k T \sqrt{B} \leftrightarrow 0^{4}$, where $k T=-174 \mathrm{dBm} / \mathrm{Hz}$ at $T=290 \mathrm{Kelvin}$. Thus, $\alpha_{U P C S}$ is independent of $\beta$ and highly dependent on bandwidth $B$. In contrast, $\alpha_{D}$ is highly dependent on $\beta$, as shown by Equation 2. As $\alpha_{U P C S}=1584 k T \sqrt{B} \leftrightarrow 0^{4}, \alpha_{U P C S}$ equals $\alpha_{D}$ when $\sqrt{B} / \beta=1.144 \leftrightarrow 0^{10}$, i.e. for $\beta \cong 8.74 \leftrightarrow 0^{-10}$ in a 100 $\mathrm{MHz}$ band. At $5 \mathrm{GHz}$ (with $46.4 \mathrm{~dB}$ loss at reference distance $1 \mathrm{~m}$ ) and a path loss modeled by a 3.0 propagation exponent, $\beta \cong 8.74 \leftrightarrow 0^{-10}$ equates to 29.6 meters between a device and its basestation. For shorter distances, we consider $\sqrt{B} / \beta=10^{7}$ that equates to 2.84 meters.

\section{References}

[1] J. M. Peha, Spectrum Management Policy Options, IEEE Communication Surveys, Fourth Quarter 1998, www.comsoc.org/pubs/surveys/4q98issue/pdf/Peha/pdf [2] FCC, Amendment of the Commissions Rules to Establish New Personal Communications Services, Memorandum Opinion and Order, FCC 94-144, Gen. Docket No. 90-314, June 9, 1994.

[3] FCC, Amendment of the Commissions Rules to Provide for Operation of Unlicensed NII Devices in the $5 \mathrm{GHz}$ Frequency Range, Report \& Order, FCC 97-005, Gen. Docket No. 96-102, Jan. 9, 1997.

[4] M. J. Marcus, Recent Progress in U.S. Millimeter Wave Spectrum Management Policy, IEEE MTT-S International Microwave Symposium Digest, vol. 2, pp. 505-7, June 1996. [5] D. G. Steer, Wireless Operation in the Unlicensed Band, Proc. Intl. Conf. on Universal Personal Communications, Sept. 1994, pp. 586-90.

[6] G. Hardin, The Tragedy of the Commons, Science, Vol. 162, 1968, pp. 1243-1248.

[7] D. P. Satapathy and J. M. Peha, Performance of Unlicensed Devices With a Spectrum Etiquette, Proceedings of IEEE Globecom, Nov. 1997, pp. 414-418.

[8] D. P. Satapathy and J. M. Peha, Etiquette Modifications For Unlicensed Spectrum: Approach and Impact," Proceedings of the 48th Annual International IEEE Vehicular Technology Conference, May 1998, vol.1, pp. 272-276. 